\title{
Prevalence and Correlations Between Temporomandibular Disorders in Dental Students Based on Diagnostic Criteria ( Dc/Tmd), Parafunctions And Psychoemotional Stress \\ Hajer Ibraheem
}

B.D.S , Msc.oral medicine(U.S.A), PHD of oral medicine

Ola Ali Khalaf

B.D.S

\begin{abstract}
The aim of the study was to assess the prevalence of temporomandibular disorders (TMD) and oral parafunctions, as well as their correlation with psychoemotional factors among dental students by using diagnostic criteria (DC/RDC).the sample's size of 407 students (169 males and 238 females) of dental college (100 fifth stage , 102 fourth stage, 99 third stage and 106 second stage ) . Firstly students subjected for stress questionnaire (perceived stress scale -10 ), secondly they subjected to different clinical and questionnaire measures according to diagnostic criteria of temporomandibular disorders DC/TMD (axis1) which have standerized series of diagnostic tests based on clinical signs and symptoms, finally the dental students subjected for oral parafunctions questionnaire using oral behavior checklist.the results obtained from this study showed that the prevalence of TMDs was significantly greater among students with oral parafunctions. We also observed that the prevalence of TMDs according to DC/TMD was higher among students with headache in this study, the prevalence of psycho-emotional stress, TMD according to DC/TMD and oral parafunctions were higher in females than males and in fifth stage greater than other stages.the prevalence of TMDs according to DC/TMD were significantly higher among females than males.
\end{abstract}

KEYWORDS

Temporomandsibular Disorders, Diagnostic Criteria ( DC/TMD ), Oral parafunctions ,Psychoemotional stress

\section{INTRODUCTION}

The temporomandibular disorders (TMD) and oral parafunctions seem to be a frequent problem inmodern societie (1). The etiopathology of the temporomandibular joints is related to muscles, teeth arches, and periodontium. Their main causes involve both pathophysiological and psychosocial factors ${ }^{(2)}$. In the literature, a significant impact of the psychoemotional factor is reported, comparable to the impact of other factors concerning physical health such as systemic diseases, malocclusions, loss of teeth, traumas, and microtraumas ${ }^{(3)}$. Stress, fatigue, anxiety, depression, sleep disorders, and a fast pace of life affect negatively the human psyche ${ }^{(4)}$. In those patients muscular related TMD is observed more often ${ }^{(5)}$.Moreover different studies report that TMD coexists with other numerous disorders such as SAPHO syndrome (synovitis, acne, pustulosis, hyperostosis, and osteitis syndrome), fibromyalgia, back- or spine ache, chronic fatigue syndrome, spastic colons, sleep disorders, congenital defects, headaches, and arthritis . Many studies report that the symptoms of the masticatory system disorders are more frequent in women than in men ${ }^{(6)}$. This may result from biological differences, including hormonal ones, and also psychosocial factors . Stallman reports that student population lives more under stress than the general population and develops considerably often TMD and oral parafunctions. The aim of this epidemiological study is to assess the prevalence of temporomandibular disorders and oral parafunctions among dental students and their correlation with psychoemotional factors by using diagnostic criteria (DC/RDC).

\section{MATERIAL AND METHODS}

The sample's size of 407(169 male and 238 females) students of 100 fifth stage , 102 fourth stage, 99 third stage and 106 second stage of college of dentistry university of Mustansiriyah in Baghdad city were included this study over the period from December 2014 to April 2015. The analysis was conducted by the diagnostic criteria for temporomandibular disorders (DC/TMD).This enables the standardization of the procedures of epidemiological studies, the unification of TMD diagnostic and exploratory criteria. The results of the study were based on the DC/TMD Axis I diagnostic criteria. Mental state of subjects was not assessed according to DC/TMD Axis II diagnoses. The students subjected to perceived stress scale -10 that identifies students with potential psycho- emotional problems. The perceived stress scale -10 which is self- administrated questionnaire. Then students were subjected to a combination of questionnaire measures and clinical examination to differentiate recommended evidence-based new DC/TMD protocol is appropriate for use in both clinical and research settings. More comprehensive instruments augment short and simple screening instruments for Axis I. These validated instruments allow for identification of patients with a range of simple to complex TMD presentations. A dual-axis Diagnostic Criteria for TMD (DC/TMD) 
will provide evidence-based criteria for the clinician to use when assessing patients, and will facilitate communication, regarding consultations, referrals, and prognosis. Finally, The students were subjected again to oral behavior checklist to determine oral parafunctions and oral habit that may have effect on the etiology of temporomandibular disorders .

\section{RESULTS}

This study revealed that most of students in the college of dentistry were under stress and the differences were significant $(p$-value $=0.044)$ between male $(41.5 \%)$ and female (58.5\%). Questionnaires are usually used together information about the prevalence of TMD in population. When students filled the specific questionnaire of DC/TMD, they revealed that students with pain have significant differences with p-value 0.023 and higher percentage was $44 \%$ in fifth class then $36 \%$ in second class, $33 \%$ in third class and $24 \%$ in fourth class. Headache recorded higher percentage than those with pain include $50 \%$ in fifth class then $27.4 \%$ in fourth class ,31.3\% in third class and $22.6 \%$ in second class and also had a highly significant differences with p-value 0.000 , while the history of pain and headache was reported higher in $17.22 \%$ females than $15.3 \%$ males. In this study, there is significant differences between clicking and genders with p-value 0.003 and females with clicking $(17.2 \%)$ recorded higher percentage than males $(13.6 \%)$ with clicking, it may result from hormonal or biological factors in addition to , stress more in females than males and it is contributing factor to TMD ., there is also significant difference between clicking and educational stages with $p$-value 0.008 and higher percentage among fifth stage (29\%) due to psychological and emotional factors then $(5.8 \%)$ in fourth stage,$(16.1 \%)$ in third stage and $(12.2 \%)$ in second stage. Statistical difference between oral parafunctions and educational stages was highly significant with p-value 0.003 and higher percentage among fifth stage was (10\%) then (4.9\%) in fourth stage,$(3.03 \%)$ in third stage and $(1.88 \%)$ in second stage.

Table 1 : Statistical Difference Between TMD and Genders
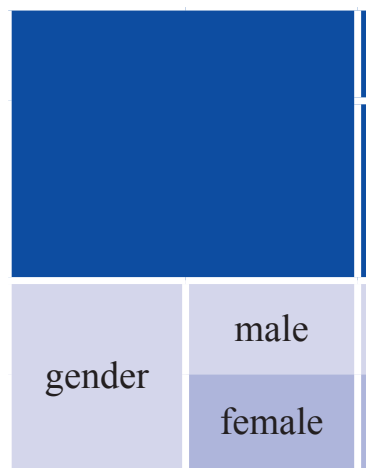

pain disorders

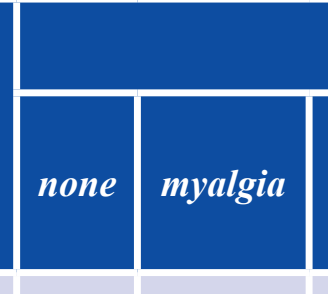

myofascial
pain with
referral

right ar-
thralgia

left ar-
thralgia

\begin{tabular}{|c|}
\hline $\begin{array}{c}\text { headache } \\
\text { attributed } \\
\text { to tmad }\end{array}$ \\
\hline 26 \\
\hline 33
\end{tabular}
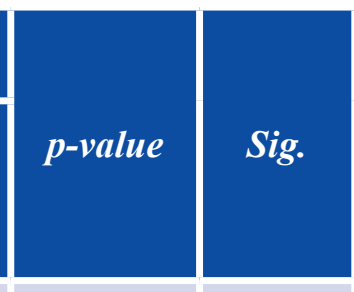

7

15

2

28

Table : Differences Between TMJ Disorders and oral parafunctions

\begin{tabular}{|c|c|c|c|c|c|c|c|c|}
\hline & & \multicolumn{5}{|c|}{ diagnoses Tmj disorders } & \multirow[b]{2}{*}{$p$-value } & \multirow[b]{2}{*}{ Sig. } \\
\hline & & $\begin{array}{c}\text { none disc } \\
\text { displacement } \\
\text { (select one) }\end{array}$ & $\begin{array}{l}\text { with reduc- } \\
\text { tion }\end{array}$ & $\begin{array}{l}\text { with reduc- } \\
\text { tion, with } \\
\text { intermittent } \\
\quad \text { locking }\end{array}$ & $\begin{array}{l}\text { without } \\
\text { reduction, } \\
\text { with limit- } \\
\text { ed opening }\end{array}$ & $\begin{array}{c}\text { without } \\
\text { reduction, } \\
\text { without } \\
\text { limited } \\
\text { opening }\end{array}$ & & \\
\hline \multirow{4}{*}{$\begin{array}{l}\text { grind teeth } \\
\text { together during } \\
\text { waking hours }\end{array}$} & YES & 36 & 10 & 8 & 3 & 0 & \multirow{4}{*}{0.000} & \multirow{3}{*}{$\mathrm{S}$} \\
\hline & $\mathrm{NO}$ & 319 & 19 & 6 & 5 & 1 & & \\
\hline & YES & 145 & 20 & 9 & 8 & 1 & & \\
\hline & NO & 210 & 9 & 5 & 0 & 0 & & \\
\hline
\end{tabular}




\section{DISCUSSION}

Temporomandibular disorders have a multifactorial etiopathogenesis. Several authors underline the influence of local factors on their development, while others underline that of systemic factors ${ }^{(7)}$. The importance of psychological factors, such as increased psychoemotional activity and stress, is also emphasized in literature describing the etiology of TMD and oral parafunctions ${ }^{(8)}$. In the presented study we also observed a significant role of psychoemotional factors in the TMD development. Easily excitable and emotionally burdened persons suffered significantly more often from TMD and oral parafunctions. It must be mentioned that the $\mathrm{DC} /$ TMD Axis I protocol includes valid diagnostic criteria for differentiating the patients in this study into two groups, with and without TMD. Although this protocol served the scope of the study, generally enables the diagnosis of a limited number and common TMD. Stress is also an important factor contributing to the TMD development. The examined student population is particularly susceptible to the influence of this factor ${ }^{(9)}$. This study revealed that most of students in the college of dentistry were under stress and the differences were significant ( $p$-value $=0.044$ ) between male $(41.5 \%)$ and female $(58.5 \%)$. We found that the female more than male in being upset with p-value 0.042 and the stressors may include a large number of duties, the pressure of getting a good education, an uncertain future, low income, living far away from home, and functioning in an alien environment.

\section{CONCLUSION}

- In all stages females showed greater prevalence of psycho-emotional stress than males

- Among students of fifth stage the prevalence of psycho-emotional stress was higher than other stages

- Oral parafunctions ( bruxism, cheek/lips biting , objects biting and nail biting ) showed greater prevalence in fifth stages than others

\section{REFERENCES}

1. S. F. Dworkin and L. LeResche, "Research diagnostic criteria for temporomandibular disorders: review, criteria, examinations and specifications, critique," Journal of Craniomandibular \& Sleep Practice, vol. 6, no. 4, pp. 301355, 1992.

2. American Society of Temporomandibular Joint Surgeons, "Guidelines for diagnosis and management of disorders involving the temporomandibular joint and related musculoskeletalstructures," Journal of CranioMaxillofacial Surgery, vol. 21, no.1, pp. 68-76, 2003.

3. M. Wie,ckiewicz, A. Paradowska, B. Kawala, and W.Więckiewicz, "SAPHO syndrome as a possible cause of masticatory system anomalies - a review of the literature,"Advances in Clinical and Experimental Medicine, vol. 20, no. 4, pp. 521-525, 2011.

4. L. R. Bonjardim, M. B. Duarte Gavĩao, L. J. Pereira, and P. M. Castelo, "Anxiety and depression in adolescents and their relationship with signs and symptoms of temporomandibular disorders," International Journal of Prosthodontics, vol. 18, no. 4, pp. 347-352, 2005.

5. L. B. Calixtre, B. L. da Silva Gr"uninger, T. C. Chaves, and A. B. de Oliveira, "Is there an association between anxiety/ depression and temporomandibular disorders in college students?" Journal of Applied Oral Science, vol. 22, no. 1, pp. 15-21, 2014.

6. A. Anastassaki K“ohler, A. Hugoson, and T.Magnusson, "Prevalence of symptoms indicative of temporomandibular disorders in adults: cross-sectional epidemiological investigations covering two decades," Acta Odontologica Scandinavica, vol. 70, no.3, pp. 213-223, 2012.

7. M. K.Murphy, R. F.MacBarb, M. E.Wong, and K. A. Anthanasiou, "Temporomandibular disorders: a review of etiology, clinical management, and tissue engineering strategies," The International Journal of Oral and Maxilofacial Implants, vol. 28, no. 6, pp. 393-414, 2013.

8. D. Manfredini, M. Marini, C. Pavan, L. Pavan, and L. Guarda- Nardini, "Psychosocial profiles of painful TMD patients," Journal of Oral Rehabilitation, vol. 36, no. 3, pp. 193-198, 2009.

9. D.Manfredini and F. Lobbezoo, "Role of psychosocial factors in the etiology of bruxism," Journal of Orofacial Pain, vol. 23, no. 2, pp. 153-166, 2009. 